\title{
Plasma osteoprotegerin levels are associated with glycaemic status, systolic blood pressure, kidney function and cardiovascular morbidity in type 1 diabetic patients
}

\author{
Lars Melholt Rasmussen, Lise Tarnow ${ }^{1}$, Troels Krarup Hansen ${ }^{2}$, Hans-Henrik Parving ${ }^{1,3}$ and Allan Flyvbjerg $^{2}$ \\ Department of Clinical Biochemistry, University Hospital of Aarhus, Aarhus Amtssygehus, T. Hansengade 2, DK-8000 Aarhus C, Denmark, ${ }^{1}$ Steno \\ Diabetes Center, Gentofte, Denmark, ${ }^{2}$ Medical Department $M$ and the Medical Research Laboratories, Aarhus University Hospital, Aarhus, Denmark and \\ ${ }^{3}$ Faculty of Health Science, University of Aarhus, Aarhus, Denmark
}

(Correspondence should be addressed to L M Rasmussen; Email: Imr@dadlnet.dk)

\begin{abstract}
Objective: The bone-related peptide osteoprotegerin (OPG) has recently been found in increased amounts in the vasculature in diabetes. It is produced by vascular smooth muscle and endothelial cells, and may be implicated in the development of vascular calcifications. OPG is present in the circulation, where increased amounts have been observed in patients with diabetes. In this study, we examined whether plasma OPG is associated with the glycaemic and vascular status of patients with type 1 diabetes.

Methods: Two gender-, age- and duration-comparable groups of type 1 diabetic patients either with $(n=199)$ or without $(n=192)$ signs of diabetic nephropathy were studied. Plasma OPG was determined by an ELISA.

Results: The plasma OPG concentration was significantly higher in patients with nephropathy than those without (3.11 (2.49-3.99) vs 2.57 (2.19-3.21) (median (interquartiles), ng/ml), $P<0.001)$. Plasma OPG correlated with haemoglobin $\mathrm{A}_{1 \mathrm{c}}\left(\mathrm{HbA}_{1 \mathrm{c}}\right)$, systolic blood pressure and age in both groups and, in addition, with kidney function in the nephropathic group. These correlations remained significant in multivariate models. In addition, we found that plasma OPG concentrations were increased among patients with cardiovascular diseases (CVD), both in the normoalbuminuric and the nephropathic groups. The differences between nephropathic and normoalbuminuric, as well as subgroups with and without CVD, could largely be ascribed to changes in $\mathrm{HbA}_{1 \mathrm{c}}$, age, systolic blood pressure and creatinine.

Conclusion: OPG is associated with glycaemic control and CVD in patients with type 1 diabetes, compatible with the hypothesis that OPG is associated with the development of diabetic vascular complications.
\end{abstract}

European Journal of Endocrinology 154 75-81

\section{Introduction}

Osteoprotegerin (OPG) is a secreted member of the tumour necrosis factor (TNF) receptor superfamily, which was initially discovered in bone where it functions as a strong anti-resorptive factor (1). It exerts its effect through binding and neutralization of the receptor activator for NF-KB ligand (RANKL), a cytokine with strong osteoclast-inducing activity. However, several observations suggest that OPG may also play important roles in the vasculature. Both experimental (2) and human studies (3) have shown that OPG is present in the arterial wall and, interestingly, genetically modified animals with deficient OPG production develop vascular media calcifications of the same type as is often observed in diabetes (2). In vitro studies have, moreover, shown that OPG is expressed in vascular smooth muscle cells $(4,5)$ and that it can act as a survival factor for endothelial cells (6). The ligand described for OPG in bone, RANKL, is not found in the vasculature under normal conditions, but may appear in calcifying areas (7); the exact role of OPG in the arterial wall is, however, currently unknown (8). Interestingly, we have recently found increased levels of OPG in arterial tissue from patients with diabetes and observed that OPG production from vascular smooth muscle cells is regulated by hormones and cytokines, like insulin and TNF- $\alpha$ (9).

OPG is present in the circulation and several studies have shown that altered concentrations can be found in relation to several bone diseases. OPG has not, however, proven to be a clear marker for either bone 
formation or bone degradation and only weak associations with bone mineral density and other parameters of bone metabolism have been reported (10). Interestingly, three independent studies have described increased plasma concentrations of OPG in patients with diabetes. In one report, high values of OPG were seen in a subgroup of type 2 diabetic women in a study primarily examining the relation between plasma OPG and bone density (11). In another investigation of 522 men, describing a positive relationship between coronary arteriosclerosis (determined by coronary arteriography) and plasma OPG, it was additionally observed that diabetic men had elevated plasma levels of OPG (12). The increase was independent of the degree of coronary sclerosis. Most recently, we reported increased plasma OPG levels in patients with type 2 diabetes, especially those with microvascular manifestations (13).

In light of the increased level of plasma OPG found in diabetes and its putative role in the genesis of diabetic macroangiopathy, we have investigated possible relations between plasma OPG and kidney function, cardiovascular disease (CVD) and glycaemic status in groups of type 1 diabetic patients with and without signs of kidney complications.

\section{Materials and methods}

\section{Subjects}

Patients from the outpatient clinic at the Steno Diabetes Center with long-standing type 1 diabetes were recruited (14). A total of 199 patients with diabetic nephropathy and 192 patients with persistent normoalbuminuria, who were comparable in relation to gender, age and duration of diabetes, were included. Diabetic nephropathy was diagnosed based on the following criteria: persistent albuminuria $>300 \mathrm{mg} / 24 \mathrm{~h}$ in at least two of three consecutive 24-h urine collections, presence of retinopathy, and no clinical or laboratory evidence of kidney or renal tract disease other than diabetic glomerulosclerosis. None of the patients was receiving treatment for osteoporosis or malignant diseases. Only a few patients received other medications (seven received immunosuppression and steroids and 17 received oestrogen treatment). The study was approved by the local ethics committee, and all participants gave their informed consent.

\section{Research design}

All investigations were performed in the morning following an overnight fast. Venous blood was drawn with minimal stasis from an antecubital vein. Centrifugation was performed within 1 hour and plasma was stored at $-80^{\circ} \mathrm{C}$.

A 12-lead electrocardiogram (ECG) was recorded and subsequently coded by two trained observers, who were blinded to the clinical status of the patients, using the Minnesota rating scale (15). Coronary heart disease (CHD) was diagnosed if the ECG showed signs of probable myocardial infarction (Minnesota rating scale 1.1-2) or possible myocardial ischaemia (Minnesota rating scale $1.3,4.1-4,5.1-3$ or 7.1 ), or if patients reported a history of either angina pectoris, defined in accordance with Rose (16), or of myocardial infarction according to World Health Organization (WHO) criteria (17). Positive manifestations of CVD were: signs of CHD, as defined above, or a history of stroke or intermittent claudication, when interviewed with the WHO cardiovascular questionnaire (17).

\section{Biochemical assays}

Urinary albumin excretion (UAE) was measured by an enzyme immunoassay (18) from 24-h urine collections. Glomerular filtration rate (GFR) was measured in patients with diabetic nephropathy after a single injection of $3.7 \mathrm{MBq}{ }^{51} \mathrm{Cr}-\mathrm{EDTA}$ by determination of radioactivity in venous blood samples taken 180, 200, 220, and $240 \mathrm{~min}$ after the injection (19). Haemoglobin $\mathrm{A}_{1 \mathrm{c}}\left(\mathrm{HbA}_{1 \mathrm{c}}\right)$ was measured by HPLC (DIAMAT; BioRad, Richmond, CA, USA) (reference range 4.1$6.1 \%)$. Serum creatinine concentration was assessed using a kinetic Jaffe method. Plasma OPG was measured by a sandwich ELISA, using commercially available antibodies (R\&D Systems, Minneapolis, MN, USA), as previously described (13). Briefly, mouse anti-human OPG (R\&D Systems) was used as capture antibody and a biotinylated goat anti-human OPG (R\&D Systems) for detection. Recombinant human OPG was used for calibration and the analytical range of the assay was $62.5-4000 \mathrm{pg} / \mathrm{ml}$. Plasma samples were diluted $1 / 3$ and measured in duplicate. The intra-assay coefficient of variation, as judged from duplicate measurements, was 3\%. The interassay variation was $8 \%(n=18$ control samples $)$ in the present series of analysis. The samples were analyzed in random order.

\section{Statistical analysis}

Plasma OPG, UAE and serum creatinine concentrations were non-normally distributed and values are given as medians with interquartile ranges in parentheses. All other values are given as means \pm S.D. For non-normally distributed variables, comparisons between groups were performed by the Mann-Whitney U test, whereas unpaired Student's $t$-test was used for normally distributed variables. Differences in frequencies were estimated by the $\chi^{2}$ test. Univariate linear relationships between variables were evaluated with Pearson's coefficient. Mean values of OPG and regression coefficients in the patient groups with and without nephropathy and CVD were adjusted for age, $\mathrm{HbA}_{1 \mathrm{c}}$, creatinine, systolic 
blood pressure, cholesterol and duration of diabetes and subsequently in linear mixed models, as indicated.

Non-normally distributed values were transformed, using natural log in these statistical procedures as indicated. All calculations were done with SPSS for Windows version 11.0 (SPSS, Chicago, IL, USA).

\section{Results}

The clinical characteristics are summarized in Table 1. Patients with nephropathy were comparable with patients with normoalbuminuria with regard to age, sex and duration of diabetes. Patients in the nephropathy group had higher $\mathrm{HbA}_{1 \mathrm{c}}(\%)$, serum creatinine $(\mu \mathrm{mol} / \mathrm{l})$, blood pressure $(\mathrm{mmHg})$ and total cholesterol (mmol/l) levels, while body mass index (BMI) was comparable in the two groups.

Plasma OPG levels $(\mathrm{ng} / \mathrm{ml})$ were statistically significantly increased in the nephropathy group, when compared with the normoalbuminuric group (Table 1). No statistically significant difference was seen in plasma OPG between men and women in each group. Using univariate analysis, we found correlations between $\ln$ (plasma OPG), $\mathrm{HbA}_{1 \mathrm{c}}$, duration of diabetes, age and systolic blood pressure in both groups (crude correlation coefficients (Pearson) as well as adjusted associations are shown in Table 2 (except for relations to the duration of diabetes, which were 0.338 and 0.359 in the normoalbuminuric and nephropathy groups respectively). Moreover, in the nephropathy group we observed significant correlations to $\ln$ (creatinine) (Table 2), UAE $(0.173, P<0.05)$ and cholesterol $(0.279, P<0.001)$. As can be seen in Fig. 1 , a strong relation was found between GFR and OPG values in the nephropathy group. Regarding the relation to blood pressure, we found, in addition, that patients treated with anti-hypertensive drugs (independent of type) had higher concentrations of OPG than those not treated with these substances $(2.53$
$(2.17-2.96)$ in untreated vs $3.23(2.52-3.76)$ in treated normoalbuminurics and $2.63(1.97-3.05)$ vs 3.31 $(2.63-4.10)$ in the nephropathy group, $P<0.001$ in both groups).

After adjusting for systolic blood pressure, cholesterol and $\mathrm{HbA}_{1 \mathrm{c}}$ in a multivariate model, as shown in Table $3, \ln$ (plasma OPG) was still significantly increased in the nephropathic patients. However when $\ln$ (creatinine) was included in the model the difference disappeared.

Manifestations of CVD were present in 84 patients, with a higher prevalence among patients with nephropathy compared with the normoalbuminuric group: $30 \%(60 / 199)$ vs $12 \%(24 / 192), \quad P<0.001$. The plasma OPG concentrations in patients with and without CVD are shown in Fig. 2. As can be seen, plasma OPG is higher in patients with CVD in both the normoalbuminuric and the nephropathic patients. When comparing different clinical variables in patients with and without CVD in both study groups, it appears that both age and $\mathrm{HbA}_{1 \mathrm{c}}$ (together with serum creatinine and systolic blood pressure in the nephropathy group) were higher in the CVD groups (Table 4 ).

Results from a multivariate analysis is shown in Table 5, where it can be seen that when the effects of age, $\mathrm{HbA}_{1 \mathrm{c}}$ and systolic blood pressure were included, the difference in OPG concentrations between patients with and without CVD disappeared in the normoalbuminuric group. Inclusion of the same variables in the nephropathy group narrowed the difference and the significant increase disappeared when $\ln$ (creatinine) was included as well, although a trend towards higher levels in patients with CVD was still present.

\section{Discussion}

In this cross-sectional study of two groups of type 1 diabetic patients with and without nephropathy, we first demonstrated that plasma OPG was higher

Table 1 Characteristics of 391 type 1 diabetic patients with either normoalbumiuria or diabetic nephropathy. Data are presented as means \pm S.D. or median (interquartile).

\begin{tabular}{|c|c|c|c|}
\hline & Normoalbuminuria & Nephropathy & $\boldsymbol{P}$ \\
\hline Number & 192 & 199 & \\
\hline Age (years) & $43 \pm 10$ & $41 \pm 10$ & NS \\
\hline $\operatorname{Sex}(M / F)$ & $118 / 74$ & $122 / 77$ & NS \\
\hline Duration of diabetes (years) & $27 \pm 9$ & $28 \pm 8$ & NS \\
\hline Daily insulin dose (IU) & $38(32-46)$ & $40(34-50)$ & $=0.046$ \\
\hline BMI $\left(\mathrm{kg} / \mathrm{m}^{2}\right)$ & $23.6 \pm 2.5$ & $24.0 \pm 3.3$ & NS \\
\hline $\mathrm{UAE}(\mathrm{mg} / 24 \mathrm{~h})$ & $8(5-13)$ & 796 (342-2079) & $<0.001$ \\
\hline Serum creatinine $(\mu \mathrm{mol} / \mathrm{l})$ & $76(70-83)$ & $103(82-134)$ & $<0.001$ \\
\hline Systolic blood pressure $(\mathrm{mmHg})$ & $132 \pm 18$ & $151 \pm 23$ & $<0.001$ \\
\hline Diastolic blood pressure $(\mathrm{mmHg})$ & $76 \pm 10$ & $86 \pm 13$ & $<0.001$ \\
\hline Serum total cholesterol $(\mathrm{mmol} / \mathrm{l})$ & $4.8 \pm 1.0$ & $5.6 \pm 1.2$ & $<0.001$ \\
\hline Retinopathy (nil/SR/PR) & $67 / 105 / 20$ & $0 / 62 / 137$ & $<0.001$ \\
\hline Plasma OPG (ng/ml) & $2.57(2.19-3.21)$ & $3.11(2.49-3.99)$ & $<0.001$ \\
\hline
\end{tabular}

NS, not significant; nil, no retinopathy; SR, simplex retinopathy; PR, proliferative retinopathy; UAE, urinary albumin excretion; BMI, body mass index. 
Table 2 Crude and adjusted associations between $\ln (\mathrm{OPG})$ and clinical parameters.

\begin{tabular}{|c|c|c|c|c|c|}
\hline & \multirow[b]{2}{*}{ Controlling for } & \multicolumn{2}{|c|}{ Normoalbuminuria } & \multicolumn{2}{|c|}{ Nephropathy } \\
\hline & & Coefficient & $P$ & Coefficient & $P$ \\
\hline \multirow{3}{*}{ Age } & - & 0.423 & $<0.001$ & 0.522 & $<0.001$ \\
\hline & Diabetes duration & 0.304 & $<0.001$ & 0.423 & $<0.001$ \\
\hline & Diabetes duration, $\mathrm{HbA}_{1 \mathrm{c}}$, In(crea), systolic BP & 0.245 & $<0.001$ & 0.362 & $<0.001$ \\
\hline \multirow[t]{3}{*}{ Systolic BP } & - & 0.315 & $<0.001$ & 0.492 & $<0.001$ \\
\hline & Age & 0.151 & 0.038 & 0.419 & $<0.001$ \\
\hline & Age, $\mathrm{HbA}_{1 \mathrm{c}}, \ln (\mathrm{crea})$ & 0.149 & 0.041 & 0.291 & $<0.001$ \\
\hline \multirow[t]{3}{*}{ In(creatinine) } & - & 0.071 & 0.890 & 0.483 & $<0.001$ \\
\hline & Age & -0.010 & 0.895 & 0.478 & $<0.001$ \\
\hline & Age, $\mathrm{HbA}_{1 \mathrm{c}}$, systolic $\mathrm{BP}$ & -0.005 & 0.944 & 0.397 & $<0.001$ \\
\hline \multirow[t]{3}{*}{$\mathrm{HbA}_{1 \mathrm{c}}$} & - & 0.215 & 0.003 & 0.218 & 0.002 \\
\hline & Age & 0.207 & 0.004 & 0.231 & 0.001 \\
\hline & Age, In(crea), systolic BP & 0.202 & 0.005 & 0.234 & 0.001 \\
\hline
\end{tabular}

BP, blood pressure; crea, creatinine.

in patients with than without diabetic nephropathy. It has previously been shown in non-diabetic individuals that serum OPG values are increased among people with impaired kidney function (20). Moreover, in that study of 46 predialysis patients with kidney failure (also including diabetics), it was observed that OPG increased with decreased kidney function, as determined by creatinine clearance. In the present study, we have found the same association between GFR and OPG levels among patients with diabetic

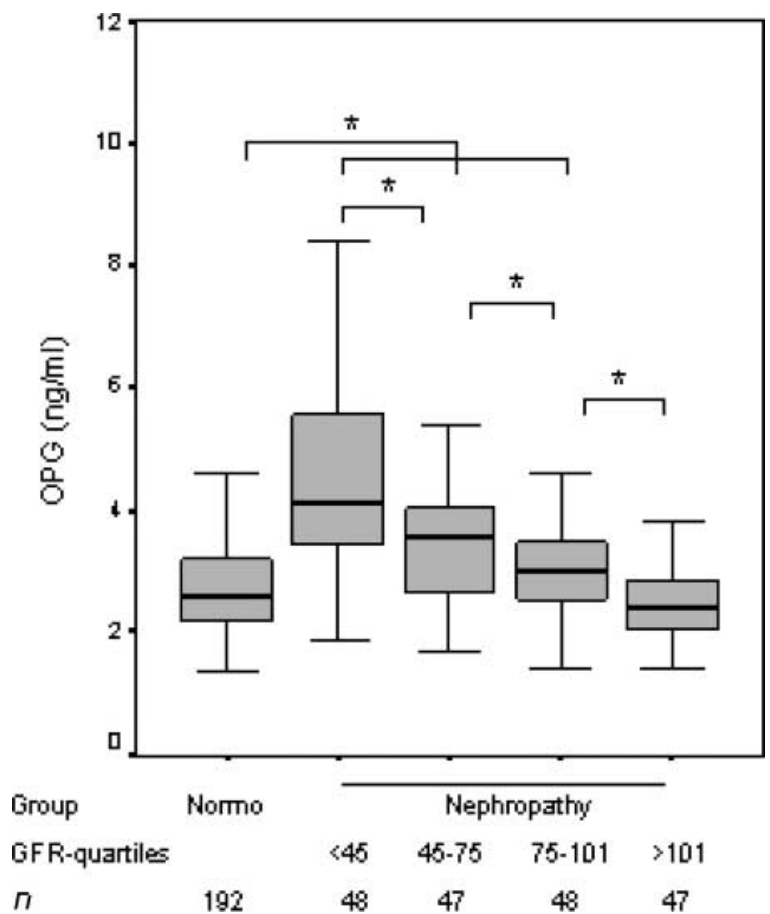

Figure 1 Plasma OPG in nephropathic patients, grouped in quartiles on the basis of GFR, and in the normoalbuminuric group (Normo). Bars represent medians and boxes indicate interquartile ranges, whereas whiskers show the 10th and 90th percentiles. ${ }^{\star} P<0.01$ between groups (Mann-Whitney $\mathrm{U}$ test). nephropathy. Although nothing is yet known about the degradation of $\mathrm{OPG}$, and the final handling of the molecule, the results are compatible with the notion that kidney excretion plays an important role in the clearance of OPG. Other explanations could relate to putative effects of metabolic, toxic or hormonal factors related to kidney failure on the metabolism of OPG. In our study, the difference between nephropathic and normoalbuminuric patients, with regard to the levels of OPG, may be at least partly due to differences in GFR, since no variation in OPG levels was observed when normoalbuminuric subjects were compared with the subgroup of nephropathic patients with GFR values above $100 \mathrm{ml} / \mathrm{min}$ per $1.73 \mathrm{~m}^{2}$. This is in line with our finding that the OPG increase in nephropathic patients disappeared when it was adjusted for changes in creatinine.

Plasma OPG levels were correlated with several factors associated with both glucose homeostasis and cardiovascular status. We previously reported a significant correlation between $\mathrm{HbA}_{1 \mathrm{c}}$ and $\mathrm{OPG}$ in a smaller group of type 2 diabetic patients (13). The present data have confirmed this observation, which was seen in both normoalbuminuric and nephropathic type 1 diabetic patients. Moreover, this result is in line with a previous study where associations between serum fructosamine and OPG were reported (11). The mechanisms behind the correlation between OPG and glycaemic status remain unknown, but may relate to possible regulatory effects of OPG production from vascular cells and osteoblasts since OPG synthesis is regulated by insulin-like growth factor-I in osteoblasts (21), by insulin and TNF- $\alpha$ in vascular smooth muscle cells (9) and by TNF- $\alpha$ in endothelial cells (22).

In addition to associations with glycaemic control, we also found significant correlates between plasma OPG levels and cardiovascular status. This was evident from the significant relations of plasma OPG to 


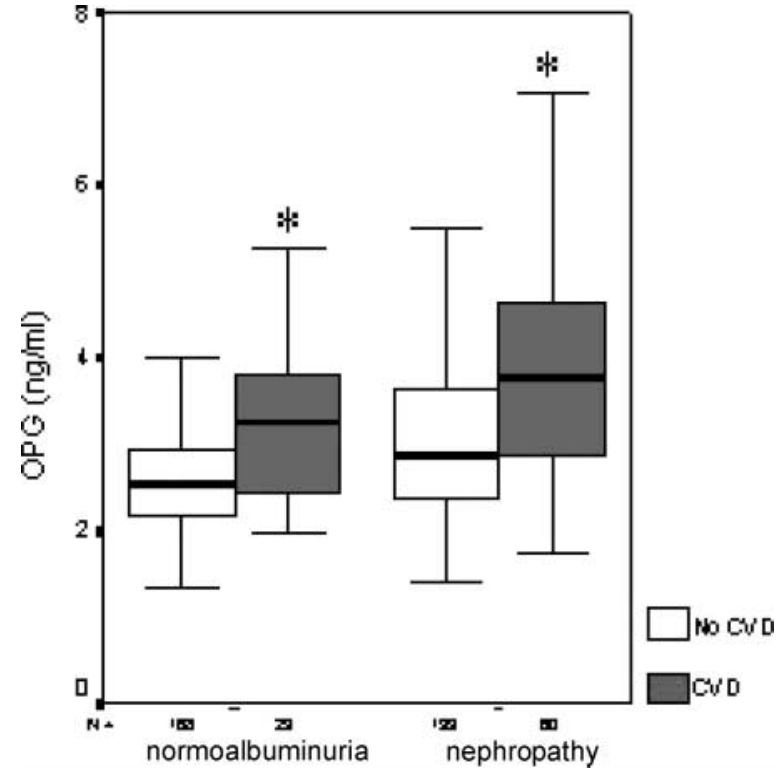

Figure 2 Plasma OPG concentrations in patients with and without signs of CVD in the normoalbuminuric and the nephropathic groups, as indicated. Bars represent medians, boxes indicate interquartile ranges and whiskers show the 10th and 90th percentiles. ${ }^{\star} P<0.01$ (Mann-Whitney $\mathrm{U}$ test). systolic blood pressure and the presence of CVD. The association between plasma OPG and systolic blood pressure has not previously been observed, but seemed relatively strong and independent of kidney function and was observed both in normoalbuminuric and nephropathic patients. In support of the correlation data, we moreover observed that patients diagnosed and medically treated for hypertension had significantly higher OPG levels than patients not treated for hypertension. The association may relate to the fact that high systolic blood pressure often coexists with increased arterial stiffness and vascular calcifications (23) and therefore may relate to the generalized matrix changes, including OPG accumulation (9) in the arterial wall in diabetes. Further strengthening the connection between plasma OPG and the vasculature was the finding of increased plasma OPG among patients with signs of CVD. This was initially observed in both patient groups but almost disappeared when adjusted for by differences in age, $\mathrm{HbA}_{1 \mathrm{c}}$ and systolic blood pressure in the normoalbuminuric group and by creatinine in the nephropathic group. Thus, the differences in OPG may at least partly relate to associations with

Table 3 Relationships between $\ln (\mathrm{OPG})$ and nephropathy. Values are estimated means (95\% confidence interval).

\begin{tabular}{lccr}
\hline Models & Normoalbuminuria & Nephropathy & $\boldsymbol{P}$ \\
\hline Crude & $0.97(0.92-1.02)$ & $1.16(1.12-1.21)$ & $<0.001$ \\
$\mathrm{HbA}_{1 \mathrm{c}}$, systolic BP, cholesterol & $1.03(0.99-1.02)$ & $1.10(1.06-1.15)$ & 0.027 \\
$\ln ($ creatinine) & $1.07(1.03-1.11)$ & $1.07(1.03-1.11)$ & 0.947 \\
\hline
\end{tabular}

$\mathrm{BP}$, blood pressure.

Table 4 Characteristics of the patients with or without CVD in both the normoalbuminuric and the nephropathic groups. Data are presented as means \pm S.D. or median (interquartile).

\begin{tabular}{|c|c|c|c|c|c|c|}
\hline & \multicolumn{3}{|c|}{ Normoalbuminuria } & \multicolumn{3}{|c|}{ Nephropathy } \\
\hline & $-C V D$ & $+C V D$ & $P$ & $-C V D$ & $+C V D$ & $P$ \\
\hline Number & 168 & 24 & & 139 & 60 & \\
\hline $\operatorname{Sex}(M / F)$ & $107 / 61$ & $11 / 13$ & NS & $87 / 52$ & $35 / 25$ & NS \\
\hline Age (years) & $42 \pm 10$ & $49 \pm 11$ & $<0.001$ & $40 \pm 9$ & $44 \pm 11$ & $<0.01$ \\
\hline $\mathrm{HbA}_{1 \mathrm{c}}(\%)$ & $8.4 \pm 1.1$ & $8.9 \pm 1.0$ & $<0.05$ & $9.4 \pm 1.5$ & $9.9 \pm 1.6$ & $=0.07$ \\
\hline Serum creatinine $(\mu \mathrm{mol} / \mathrm{l})$ & $76(70-82)$ & $78(71-87)$ & NS & $97(79-121)$ & $117(88-199)$ & $<0.001$ \\
\hline Serum total cholesterol $(\mathrm{mmol} / \mathrm{l})$ & $4.7 \pm 1.0$ & $5.0 \pm 1.0$ & NS & $5.5 \pm 1.2$ & $5.9 \pm 1.3$ & NS \\
\hline Systolic BP $(\mathrm{mmHg})$ & $131 \pm 17$ & $138 \pm 27$ & NS & $148 \pm 22$ & $158 \pm 23$ & $<0.01$ \\
\hline Diastolic BP $(\mathrm{mmHg})$ & $76 \pm 10$ & $74 \pm 9$ & NS & $86 \pm 13$ & $86 \pm 12$ & NS \\
\hline
\end{tabular}

NS, not significant; BP, blood pressure.

Table 5 Relationships between $\ln (\mathrm{OPG})$ and CVD in diabetic patients with and without nephropathy. Values are estimated means (95\% confidence intervals).

\begin{tabular}{|c|c|c|c|c|c|c|}
\hline \multirow[b]{2}{*}{ Models } & \multicolumn{3}{|c|}{ Normoalbuminuria } & \multicolumn{3}{|c|}{ Nephropathy } \\
\hline & $-C V D$ & $+\mathrm{CVD}$ & $P$ & $-C V D$ & $+\mathrm{CVD}$ & $P$ \\
\hline Crude & $0.95(0.91-0.99)$ & $1.13(1.01-1.24)$ & $<0.001$ & $1.08(1.02-1.14)$ & $1.34(1.25-1.43)$ & $<0.001$ \\
\hline $\mathrm{HbA}_{1 \mathrm{c}}$, systolic $\mathrm{BP}$ & $0.96(0.93-1.00)$ & $1.02(0.92-1.13)$ & 0.30 & $1.13(1.08-1.17)$ & $1.24(1.17-1.31)$ & 0.011 \\
\hline In(creatinine) & $0.96(0.93-1.00)$ & $1.02(0.92-1.13)$ & 0.30 & $1.14(1.10-1.18)$ & $1.20(1.13-1.27)$ & 0.18 \\
\hline
\end{tabular}

BP, blood pressure. 
well-known risk variables. The results concerning signs of CVD are compatible with observations in non-diabetics, where plasma OPG in two different reports was found to be associated with the severity of coronary artery disease, as determined by coronary angiography $(12,24)$. In contrast to the present data, two previous papers have reported higher OPG levels in women than men in the same age group, as studied in the present study $(25,26)$. Whether this discrepancy may relate to the particularly increased risk of CVD in women among diabetics (27) remains to be studied.

Clearly, the explanation behind the differences in OPG levels among patients with signs of CVD is multifaceted and it seems worth considering that OPG is present in the arterial wall, and moreover is found in increased amounts in diabetes (9) where it may relate to the development of vascular calcifications $(2,3)$, a phenomenon often seen in diabetes (28). Also, it is interesting that OPG is produced from vascular smooth muscle cells (5) modifiable by insulin and TNF- $\alpha$ (9) and that OPG may serve as a survival factor for endothelial cells (6). The prominent association between age and plasma OPG, as observed in the present study, has previously been seen among non-diabetics $(10,20)$. It has been described in detail in a study comprising a large number of people (10), where OPG concentrations were found to increase with age. The increase was, however, first observed above the age of 60 years among women and 70 years among men. The average age of the individuals in the present study was considerably lower and, accordingly, it is interesting that the correlation was demonstrated in type 1 diabetic patients at a lower age, and that it was dominant in particular among patients with nephropathy. The mechanisms responsible for the association between OPG and age are unknown, but alterations in bone metabolism with age have been suggested (10). Our study may, however, indicate that the relation to age also involves mechanisms related to both glucose homeostasis and vascular biology.

In conclusion, increased plasma OPG was observed in subgroups of type 1 diabetic patients with nephropathy and with signs of CVD. In addition, we observed independent positive correlations between plasma OPG and kidney function, $\mathrm{HbA}_{1 \mathrm{c}}$ and systolic blood pressure. The associations between OPG and both glycaemic and cardiovascular status in patients with diabetes are multifaceted but may relate to the involvement of OPG in the development of diabetic vascular complications.

\section{Acknowledgements}

We thank Kirsten Hald for invaluable technical assistance. The Danish Heart Foundation, the Danish Diabetes Foundation and the Danish Medical Research Foundation supported the study.

\section{References}

1 Simonet WS, Lacey DL, Dunstan CR, Kelley M, Chang MS, Luthy R, Nguyen HQ, Wooden S, Bennett L, Boone T, Shimamoto G, DeRose M, Elliott R, Colombero A, Tan HL, Trail G, Sullivan J, Davy E, Bucay N, Renshaw-Gegg L, Hughes TM, Hill D, Pattison W, Campbell P, Sander S, Tarpley J, Derby P, Lee R \& Boyle WJ. Osteoprotegerin: a novel secreted protein involved in the regulation of bone density. Cell $1997 \mathbf{8 9}$ $309-319$.

2 Bucay N, Sarosi I, Dunstan CR, Morony S, Tarpley J, Capparelli C, Scully S, Tan HL, Xu W, Lacey DL, Boyle WJ \& Simonet WS. Osteoprotegerin-deficient mice develop early onset osteoporosis and arterial calcification. Genes and Development $1998 \mathbf{1 2}$ $1260-1268$.

3 Tyson KL, Reynolds JL, McNair R, Zhang Q, Weissberg PL \& Shanahan CM. Osteo/chondrocytic transcription factors and their target genes exhibit distinct patterns of expression in human arterial calcification. Arteriosclerosis, Thrombosis, and Vascular Biology 200323 489-494.

4 Fu M, Zhang J, Lin YY, Zhu X, Willson TM \& Chen YE. Activation of peroxisome proliferator-activated receptor gamma inhibits osteoprotegerin gene expression in human aortic smooth muscle cells. Biochemical and Biophysical Research Communications 2002294 597-601.

5 Zhang J, Fu M, Myles D, Zhu X, Du J, Cao X \& Chen YE. PDGF induces osteoprotegerin expression in vascular smooth muscle cells by multiple signal pathways. FEBS Letters $2002 \mathbf{5 2 1}$ $180-184$.

6 Malyankar UM, Scatena M, Suchland KL, Yun TJ, Clark EA \& Giachelli CM. Osteoprotegerin is an alpha vbeta 3-induced, NF-kappa B-dependent survival factor for endothelial cells. Journal of Biological Chemistry 2000275 20959-20962.

7 Schoppet M, Al Fakhri N, Franke FE, Katz N, Barth PJ, Maisch B, Preissner KT \& Hofbauer LC. Localization of osteoprotegerin, tumor necrosis factor-related apoptosis-inducing ligand, and receptor activator of nuclear factor-kappaB ligand in Monckeberg's sclerosis and atherosclerosis. Journal of Clinical Endocrinology and Metabolism 200489 4104-4112.

8 Collin-Osdoby P. Regulation of vascular calcification by osteoclast regulatory factors RANKL and osteoprotegerin. Circulation Research 200495 1046-1057.

9 Olesen P, Ledet T \& Rasmussen LM. Arterial osteoprotegerin: increased amounts in diabetes and modifiable synthesis from vascular smooth muscle cells by insulin and TNF-alpha. Diabetologia $200548561-568$.

10 Kudlacek S, Schneider B, Woloszczuk W, Pietschmann P \& Willvonseder R. Serum levels of osteoprotegerin increase with age in a healthy adult population. Bone 200332 681-686.

11 Browner WS, Lui LY \& Cummings SR. Associations of serum osteoprotegerin levels with diabetes, stroke, bone density, fractures, and mortality in elderly women. Journal of Clinical Endocrinology and Metabolism 200186 631-637.

12 Schoppet M, Sattler AM, Schaefer JR, Herzum M, Maisch B \& Hofbauer LC. Increased osteoprotegerin serum levels in men with coronary artery disease. Journal of Clinical Endocrinology and Metabolism $2003 \mathbf{8 8} 1024-1028$.

13 Knudsen ST, Foss CH, Poulsen PL, Andersen NH, Mogensen CE \& Rasmussen LM. Increased plasma concentrations of osteoprotegerin in type 2 diabetic patients with microvascular complications. European Journal of Endocrinology 2003 149 39-42.

14 Tarnow L, Cambien F, Rossing P, Nielsen FS, Hansen BV, Lecerf L, Poirier O, Danilov S \& Parving HH. Lack of relationship between an insertion/deletion polymorphism in the angiotensin I-converting enzyme gene and diabetic nephropathy and proliferative retinopathy in IDDM patients. Diabetes $1995 \mathbf{4 4} 489-494$.

15 Blackburn H, Keys A, Simonson E, Rautaharju P \& Punsar S. The electrocardiogram in population studies. A classification system. Circulation 196021 1160-1175. 
16 Rose GA. The diagnosis of ischaemic heart pain and intermittent claudication in field surveys. Bulletin of World Health Organisation $196227645-658$.

17 Rose GA \& Blackburn H. Cardiovascular survey methods. Monogram Series of World Health Organisation $1968561-188$.

18 Feldt-Rasmussen B, Dinesen B \& Deckert M. Enzyme immunoassay: an improved determination of urinary albumin in diabetics with incipient nephropathy. Scandinavian Journal of Clinical and Laboratory Investigations $1985 \mathbf{4 5} 539-544$.

19 Mortensen JB \& Rodbro P. Comparison between total and renal plasma clearance of [51Cr] EDTA. Scandinavian Journal of Clinical and Laboratory Investigations $197636247-249$.

20 Kazama JJ, Shigematsu T, Yano K, Tsuda E, Miura M, Iwasaki Y, Kawaguchi Y, Gejyo F, Kurokawa K \& Fukagawa M. Increased circulating levels of osteoclastogenesis inhibitory factor (osteoprotegerin) in patients with chronic renal failure. American Journal of Kidney Diseases 200239 525-532.

21 Rubin J, Ackert-Bicknell CL, Zhu L, Fan X, Murphy TC, Nanes MS, Marcus R, Holloway L, Beamer WG \& Rosen CJ. IGF-I regulates osteoprotegerin (OPG) and receptor activator of nuclear factorkappaB ligand in vitro and OPG in vivo. Journal of Clinical Endocrinology and Metabolism $2002874273-4279$.

22 Kim HH, Shin HS, Kwak HJ, Ahn KY, Kim JH, Lee HJ, Lee MS, Lee ZH \& Koh GY. RANKL regulates endothelial cell survival through the phosphatidylinositol 3(-kinase/Akt signal transduction pathway. FASEB Journal 200317 2163-2165.
23 Benetos A, Waeber B, Izzo J, Mitchell G, Resnick L, Asmar R \& Safar M. Influence of age, risk factors, and cardiovascular and renal disease on arterial stiffness: clinical applications. American Journal of Hypertension 200215 1101-1108.

24 Jono S, Ikari Y, Shioi A, Mori K, Miki T, Hara K \& Nishizawa Y. Serum osteoprotegerin levels are associated with the presence and severity of coronary artery disease. Circulation $2002 \mathbf{1 0 6}$ 1192-1194.

25 Khosla S, Arrighi HM, Melton LJ, Atkinson EJ, O'Fallon WM, Dunstan C \& Riggs BL. Correlates of osteoprotegerin levels in women and men. Osteoporosis International 200213 394-399.

26 Bernstein CN, Sargent M \& Leslie WD. Serum osteoprotegerin is increased in Crohn's disease: a population-based case control study. Inflammatory Bowel Disease 200511 325-330.

27 Resnick HE \& Howard BV. Diabetes and cardiovascular disease. Annual Review of Medicine 200253 245-267.

28 Lehto S, Niskanen L, Suhonen M, Ronnemaa T \& Laakso M. Medial artery calcification. A neglected harbinger of cardiovascular complications in non-insulin-dependent diabetes mellitus. Arteriosclerosis, Thrombosis, and Vascular Biology $1996 \mathbf{1 6}$ 978-983.

Received 16 June 2005

Accepted 13 September 2005 